\title{
Zigbee Wireless Sensor Network Nodes Deployment Strategy for Digital Agricultural Data Acquisition
}

\author{
Xinjian Xiang ${ }^{1, *}$ and Xiaoqing Guo ${ }^{2}$ \\ ${ }^{1}$ School of Automation and Electrical Engineering, Zhejiang University of Science and \\ Technology, Hangzhou, Zhejiang Province, P.R. China 310023 \\ Tel.: 0571-85070268 \\ hzxxj@sina.com \\ ${ }^{2}$ School of Biological and Chemical Engineering, Zhejiang University of Science and \\ Technology, Hangzhou, Zhejiang Province, P.R. China 310023
}

\begin{abstract}
ZigBee is an emerging wireless network technology, according to china's digital agricultural feature such as remote, dispersion, variability and diversity, the ZigBee-based wireless sensor network for digital agricultural data acquisition is one of the best ways to build the system. In this paper, based on ZigBee wireless sensor network deployment planning principles and the status of our digital agriculture, we study several ZigBee wireless sensor network nodes deployment program for different condition and calculated their largest network capacity, network latency and other parameters, finally proposed a wireless sensor network nodes deployment strategy for digital agricultural data acquisition. This strategy is no specific requirements to the wireless sensor network topology structure, and can support the ZigBee wireless sensor network by random and manual deployment.
\end{abstract}

Keywords: Digital agricultural data acquisition, zigbee wireless sensor network, node deployment network planning; capacity calculation.

\section{Introduction}

China's agriculture has characters such as geographically dispersed, diverse objects, biological variation, and uncertain environmental factors, also the most obvious areas affect by environment, it's essential for digital agricultural data acquisition (Wei Quan et al., 2008). Existing data acquisition system use manual or pre-wiring cable acquisition. Those methods increase the work and can not keep real-time effective data acquisition; they have obvious limitations affected by geographical location, physical lines and the complexity of environmental factors. Modern wireless network technology and computer application develop fast in several years, it is necessary to combine the last technology with the area of agriculture. According to china's digital agricultural feature such as remote, dispersion, variability and diversity, the ZigBeebased wireless sensor network for digital agricultural data acquisition is one of the best ways to build the system (Lu Zhao quan et al., 2008).

\footnotetext{
* Corresponding author.
} 
Wireless sensor network is consist of many micro-sensor nodes deployed in the monitoring region. Through wireless communication, a form of self-organizing multihop network, which aims at collecting objects' information without topographical constraints and send to control center (Tian Wang-lan et al., 2008). Zigbee wireless technology with a small size, low power feature, therefore, the establishment of a ZigBee-based wireless sensor network technology can meet these requirements. In this paper, based on ZigBee wireless sensor network deployment planning principles and the status of our digital agriculture, we study several ZigBee wireless sensor network nodes deployment program for different condition and calculated their largest network capacity, network latency and other parameters, finally proposed a wireless sensor network nodes deployment strategy for digital agricultural data acquisition.

\section{ZigBee Technology Introduction}

ZigBee is a new kind of short-distance, low-power, low data transfer rate, low cost, low complexity wireless network technology, is a communication solution between wireless markup technology and Bluetooth technology, mainly used for close wireless connections. Zigbee connect and communicate among thousands of tiny sensor. These sensors require very little energy to send data from one sensor to another sensor through radio waves in a relay way, and the communication efficiency is very high (Ban Yanli et al., 2007).

The foundation of ZigBee is the IEEE 802.15.4 agreement, which is consist of PHY and MAC layer based on IEEE 802.15.4 and the network and application support layer of ZigBee. The network system has low power consumption, low cost, short delay time, high capacity, safety features. IEEE 802.15.4 agreement clearly defines the three kinds of topologies: star structure, cluster-like structure and network structure. Agreement defines two kinds of physical equipment used mutually, named the full function equipment (FFD) and simplify function equipment (RFD):

The full function equipment support any kind of topology structure, and can communicate with any equipment; simplified function equipment only support star structure, and can only communicate with the full function equipment (FFD). IEEE 802.15.4 network need one full function equipment as network consultation, and terminal node generally use simplified function equipment (RFD) to reduce the system cost and improve power battery life. In ZigBee network layer, the distributed address scheme is used to distribute network address. This scheme distributes a limited network address for the equipment, and these addresses are the unique in a particular network. ZigBee Coordinator is a full-function device, which decided the greatest number of sub-equipment to connect its network.

\section{Zigbee Wireless Sensor Network Node Deployment in Digital Agricultural Data Acquisition}

Before the design, it is necessary to know product application environment and Zigbee characteristic well. Application environment includes data acquisition structure, wireless channel and transmission environment, and product features include RF output power and receiver sensitivity. 
Network capacity and latency should be considered in general node deployment. Standard ZigBee network capacity can support up to 65,000 network nodes, each two adjacent nodes need $15 \mathrm{~ms}$ to complete a communication. However, in practical applications need to consider network coverage and response time. Node capacity is proportional to network coverage, and system doesn't with long response time. This requires design a different network topology structure for different application environment. Design of network capacity and node deployment in different network topology structure under ideal conditions as follows.

\subsection{Space Structure of Digital Agriculture Data Acquisition}

China's agriculture has characters such as geographically dispersed, diverse objects, biological variation, and uncertain environmental factors. According to China's topography, climate truck characteristics and the regional agricultural economic development in different ways, agricultural production area can be plain, high slope, hills, grasslands, mountains, lakes, wetlands and so on, corresponding to the respective sites of agricultural production is farmland, orchards, gardens, pastures, fish ponds, fisheries, etc. Based on the principle of ZigBee networking, the structure of digital agricultural data acquisition as followed:

(1) Linear structure. Such as small rectangular greenhouses, slender shape fish ponds.

(2) Planar network structure. Such as a square canopy, large-scale farmland, pasture, etc...

(3) Space network structure. Such as orchards, gardens in the mountains and so on.

(4) Mixed structure. It is a cyberspace with linear, planar network and space network structure. Such as large-scale farm include fish ponds, farms, and orchards.

\subsection{Zigbee Wireless Sensor Network Nodes Deployment with Linear Structure}

Linear structure network is a simply network can be used in rectangular greenhouses' digital agricultural data acquisition. The entire network, only a single path, which determines the number of nodes equal to network layers, or hop (Hop). The central node located in the center of the entire network, network scanning cycle (the time central node collecting network data form all the backbone nodes) directly depend on the network hops, or backbone nodes. Every communication cycle for the calculation of $15 \mathrm{~ms}$, then the entire network scanning cycle $\mathrm{T}$ can be expressed as:

$$
\mathrm{T}=15 * 2 *(1+2+3+\cdots n)
$$

Where: $\mathrm{T}$ for the entire network scan cycle $(\mathrm{ms})$; $\mathrm{n}$ for the network layer, and the network nodes is $2 \mathrm{n}$. When $\mathrm{T}=30 \mathrm{~s}$, the calculation of available $\mathrm{n}=43$, that is, linear structure network has maximum capacity of 86 nodes in the 30 s scanning cycle. Communication distance of $100 \mathrm{~m}(0.1 \mathrm{~km})$ calculated, then the coverage of the entire network for $(\mathrm{n}-1) * 2 * 100=8.4 \mathrm{~km}$ long linear region. Based on the above formula, when $\mathrm{n}=22, \mathrm{~T}=5 \mathrm{~s}$, that is, network can be divided into six slip in the 30s scanning cycle, each slip has maximum capacity of 44 nodes, this will allow network coverage up to $14 \mathrm{~km} 2$ (approximate $\pi \mathrm{r} 2=13.85$ ), the entire network capacity also increased to 264 nodes. 
Analysis of Conclusions: a single slip of linear network has maximum capacity of 86 nodes in the 30s scanning cycle, and to minimize hops help increase network capacity.

\subsection{Zigbee Wireless Sensor Network Nodes Deployment with Planar Network Structure}

Planar network structure such as large-scale farmland, pasture is complex, due to the multi-path network; scanning time analysis is also more complicated, this paper takes square instead of round for a brief analysis.

ZigBee planar network structure diagram as shown in Fig.1, assuming set a node at any two straight lines' cross-point, and central node at the center of network, there are 8 nodes( which around the central node ) can communicate directly (1 hop), 16 nodes for 2 hop and 24 nodes for 3 hop. The whole network scan time can be expressed as:

$$
\begin{gathered}
\mathrm{T}=15 \mathrm{~ms} * 8 *(1+4+9+\cdots+\mathrm{n} * \mathrm{n}) \\
\mathrm{N}=8 *(1+2+3+\cdots+\mathrm{n})
\end{gathered}
$$

Where: $\mathrm{n}$ for the network layer; $\mathrm{N}$ for the network nodes' capacity.

When $\mathrm{T}=30 \mathrm{~s}$, the calculation of available $\mathrm{n}=8, \mathrm{~N}=268$, that is, planar network has maximum capacity of 268 nodes in the 30 s scanning cycle. Communication distance of $100 \mathrm{~m}(0.1 \mathrm{~km})$ calculated, and then the coverage of the entire network is $1.6 *$ $1.6=2.56 \mathrm{~km} 2$ area. If the central node at the edge of the entire network topology, it is clear that the network will increase the number of layers, extend the scanning time and reduce the network nodes' capacity.

Analysis of Conclusions: The central node should set at the center of the network topology as much as possible, the more close to the edge, the longer scanning time, and in the limited scan time, the whole capacity of the network will become even smaller.

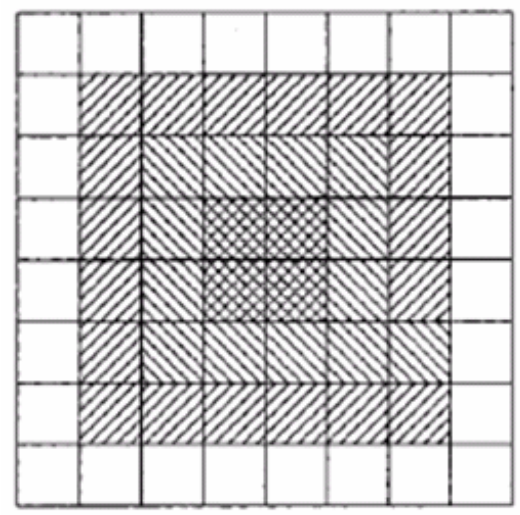

Fig. 1. ZigBee planar network structure 


\subsection{Zigbee Wireless Sensor Network Nodes Deployment with Space Network Structure}

Space network structure is more complex. It is conceivable that in the N-storey orchard, each storey with $\mathrm{n}$ blocks of terrace set one node. In this structure, the whole network scans time as follows:

$$
\mathrm{T}=\mathrm{N} * \mathrm{t}+\mathrm{n} * 15 \mathrm{~ms}(1+2+3+\cdots+\mathrm{N}-1)
$$

Where: $\mathrm{T}$ for the entire network scanning cycle; $\mathrm{t}$ for single-storey scan cycle; $\mathrm{n}$ is the number of nodes on each plane; $\mathrm{N}$ for the space layers. Based on the above formula, each plane can be treated as a planar network structure, and all of these make the space network structure. In 30s scanning cycle, it is no more than 11 layers when each layer has 25 nodes. If treat each plane as a two-branch linear network structure, it is no more than 8 layers when each layer has 24 nodes.

Analysis of Conclusions: The system performance of space network structure is relate to each layers' node number. In order to increase the network capacity, every layer's node number should be limited.

\section{Conclusion}

Zigbee network nodes deployment strategy is very important in the process of build digital agricultural data acquisition network. In order to reduce the workload of optimize network deployment, and build flexible network, the network topology structure should be decided by the environment. Network deployment is a systematic project, need for a wealth experience of wireless network planning and accumulation.

\section{Acknowledgements}

This material is based upon work funded by Zhejiang Provincial Natural Science Foundation of China under Grant No. Y108268.

\section{References}

Rui, W.Q.H., Qiang, J.: Industrial Control Network Model Based on ZigBee. Mathematics In Practice and Theory 15, 174-177 (2008)

Zhao quan, L., et al.: Greenhouse Planting Temperature Monitoring System Based on ZigBee Wireless Network. Journal of Anhui Agri. Sci. 36(13), 5682-5684 (2008)

Wang-lan, T.: Studying of the Factor that Affects the Energy Consume in the Wireless Sensor Network. Computer Knowledge and Technology 4, 742-744 (2008)

Yanli, B., Qiaolin, C., Chen, W.: ZigBee tree routing algorithm based on energy balance. Computer Applications 11, 2791-2794 (2008) 